\title{
Rare cardiovascular anomaly: congenital unilateral absence of the pulmonary artery
}

\author{
Bogusław Mazurek ${ }^{1}$, Lesław Szydłowski ${ }^{1}$, Magdalena Mazurek¹, Joanna Kohut', Jan Głowacki²
}

1Department of Pediatric Cardiology, Medical University of Silesia, Katowice, Poland 2Radiology Department, Medical University of Silesia, Silesian Centre for Heart Diseases, Zabrze, Poland

Submitted: 29 March 2011

Accepted: 19 October 2011

Arch Med Sci 2013; 9, 6: 1162-1164

DOI: 10.5114 /aoms.2013.37011

Copyright (c) 2013 Termedia \& Banach

Congenital unilateral absence of the pulmonary artery (UAPA) is an extremely rare heart defect (approximately $0.39 \%$ of all congenital heart diseases). It is frequently accompanied by other heart diseases such as tetralogy of Fallot, septal defects, aortic coarctation, subvalvular aortic stenosis, transposition of the great arteries or pulmonary stenosis. This defect also occurs in an isolated form with no other intra- or extracardiac pathologies, and it may concern the proximal segment of the left or right pulmonary artery [1-3]. The absence of pulmonary artery may also occur concomitantly with pulmonary hypoplasia or pulmonary vein anomalies [4]. The embryological cause of pulmonary artery absence is resorption of the proximal segment of the sixth aortic arch. The lungs are then supplied by the system of collateral arteries from bronchial, intercostal, subclavian and even coronary arteries [5]. The increased pressure in the collateral arteries may lead to many lesions, damage of pulmonary arteries, endothelial tissue impairment and pulmonary hypertension development [6].

In this paper, we present two case reports of patients hospitalized in the Department of Pediatric Cardiology at the Medical University of Silesia in Katowice and diagnosed with the congenital absence of the left branch of the pulmonary artery.

A 12-year-old male patient was admitted to the hospital for suspected hypoplasia of the left pulmonary artery. His medical history revealed rehabilitation due to a chest wall deformity (collapse of the right chest wall) since the age of 1.5 years which was the reason for chest $X$-rays to be performed. It showed a reduced left lung volume and poorly seen left pulmonary arteries. The patient led a normal life, experienced no frequent diseases and participated in PE lessons. On admission, his general condition was good; weight $38 \mathrm{~kg}(25 \mathrm{pc})$; height $150 \mathrm{~cm}$ (25-50 pc). Laboratory results, including gasometry, were within normal limits. The echocardiogram revealed absence of the left branch of the pulmonary artery, while the right pulmonary artery was dilated with normal flow and no pulmonary hypertension signs. The aortic arch was positioned in midline. Also, a computed tomography (CT) angiogram of pulmonary arteries was performed (Figure 1). It showed atresia of the left pulmonary artery with no additional supply to the left lung directly from the aorta. Furthermore, an aortic arch anomaly in the form of an incomplete vascular ring was observed, with the dull-ended left aortic arch branching off to the left common carotid artery and left subclavian artery as well as the right aortic arch branching off to the right common carotid artery and right subclavian artery. Between
Corresponding author:

Bogusław Mazurek MD, PhD

Department

of Pediatric Cardiology

Medical University of Silesia

16 Medykow St

40-752 Katowice, Poland

Phone: +48 322071855 ,

+48322071858

Fax: +48 322071854

E-mail: mazurek30@op.pl 
the arches, the trachea was positioned with no signs of direct compression. After a cardiosurgical consultation, the patient was qualified for further observation. He is being followed up at the outpatient cardiac clinic and reports no medical problems.

A 14-month-old male patient (staying at a children's home; frequently hospitalized due to bronchitis and pneumonia; currently presenting symptoms of atopic eczema; in good general condition) was admitted to the hospital for a suspected atrial septal defect. The physical examination revealed weight deficiency (current weight $7300 \mathrm{~g},<3 \mathrm{pc}$ ) and psychomotor retardation. Laboratory and gasometry (95-98\%) results as well as the electrocardiogram were normal. The echocardiogram did not confirm a structural intracardiac defect, but absence of the left pulmonary artery was observed. A CT angiogram of the pulmonary arteries (Figure 2) revealed absence of the proximal segment of the left pulmonary artery. Peripherally, within up to $15 \mathrm{~mm}$, the distal segment of the left pulmonary artery (probably the inferior lobar branch) was seen, supplied by at least two major aorto-pulmonary collateral arteries from the descending aorta. Furthermore, the right-sided aortic arch was found. The patient is being followed up at the out-patient cardiac clinic. He still experiences frequent respiratory infections.

Congenital unilateral absence of the pulmonary artery is more often right-sided (63\% according to Ten Harkel) and as such, it is typically an isolated case. Absence of the left branch is more frequently accompanied by other heart defects [2]. Bouros et al. estimate that $75 \%$ of patients with no left

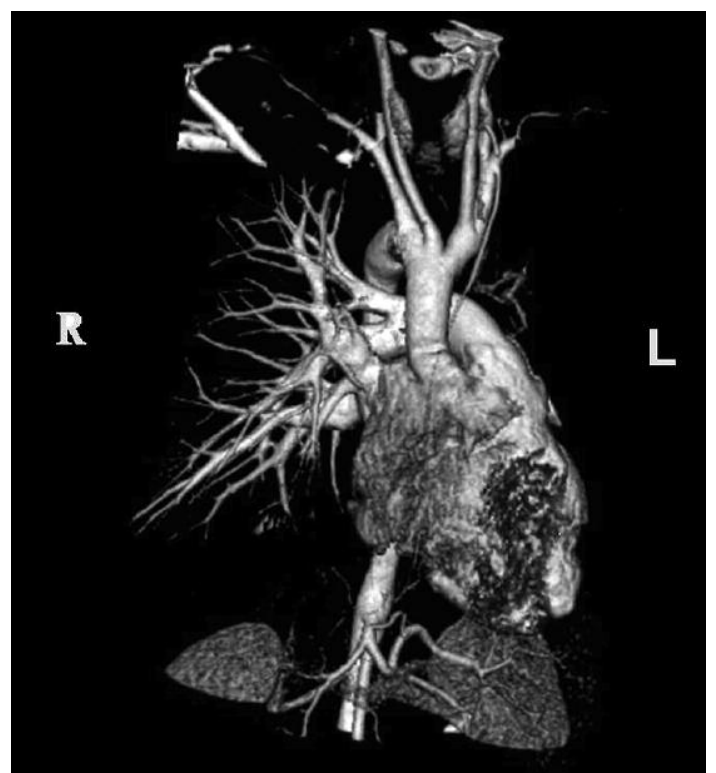

Figure 1. Case I. CT angiogram. Absence of the proximal part of the left pulmonary artery. Right-sided aortic arch branch of the pulmonary artery suffer from concomitant heart diseases [1]. In both case reports, the absence regarded the left branch of the pulmonary artery, yet without concomitant intracardiac defects except for the aortic arch anomaly in the first case, which was not manifested by any clinical symptoms requiring therapy.

In patients with concomitant heart defects, UAPA is diagnosed relatively quickly. However, if the anomaly is isolated, observed symptoms are usually non-specific: mostly respiratory infections, lower exercise tolerance or dyspnea [2, 4]. One of the reported patients experienced very frequent respiratory infections requiring intensive pharmacotherapy and kinesitherapy. The other boy, however, did not experience any diseases more frequently than his peers and his development was normal, with a lifestyle typical for his age. In his case, the reason for performing diagnostic tests was the chest wall deformity manifested by collapse of the right, thus healthy, side.

In many patients with congenital absence of the pulmonary artery, nagging chest pain or pleural effusion occurs. In the literature, the incidence of these symptoms is estimated to be approximately $37 \%$. Due to collateral circulation, $10 \%$ to $20 \%$ of patients experience hemoptysis [2, 7] or even hemorrhages which may be fatal. The signs of severe pulmonary hypertension are relatively frequent (in $25 \%$ of patients) and they usually concern the contralateral lung. Episodes of circulatory failure have also been reported. Manifestation of these serious complications may be triggered by additional factors such as pregnancy or altitude sickness. Patients with the isolated anomaly may also be asympto-

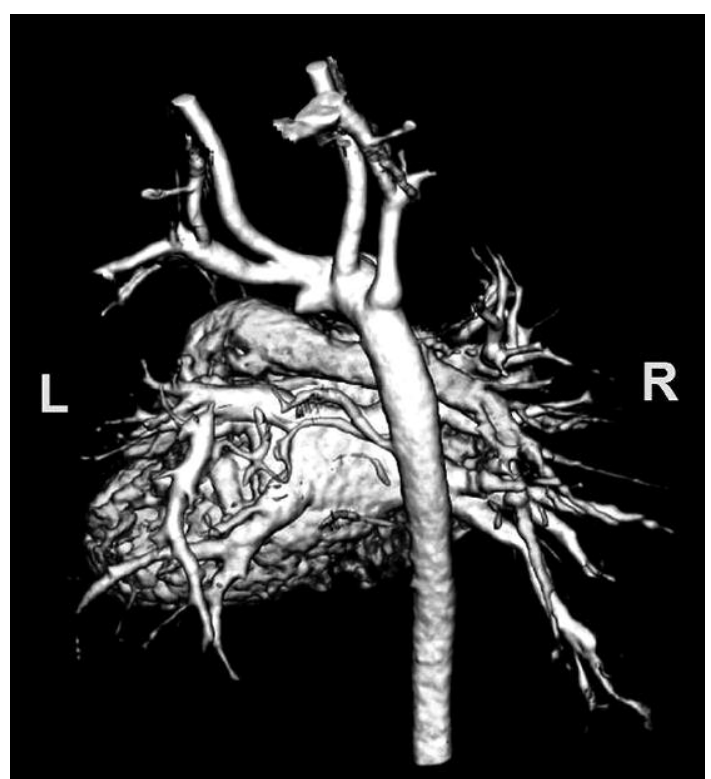

Figure 2. Case II. CT angiogram. Absence of the left pulmonary artery. Double aortic arch - incomplete vascular ring 
matic for many years and diagnosed incidentally. Nevertheless, intensity of symptoms, especially warning ones such as hemoptysis, respiratory hemorrhage or pulmonary hypertension, increases with age.

A thorough medical history (frequent infections) as well as a chest X-ray that reveals the absence of hilar contours, diminished pulmonary vascular pattern, mediastinal shift to the diseased side or pleural effusion may help determine a diagnosis [8]. In the reported patients, the analogical features were shown in radiograms. An extremely valuable diagnostic modality is an echocardiogram, which may contribute to the diagnosis or suspected diagnosis of the pulmonary artery branch absence and the assessment of potential pulmonary hypertension. Due to susceptibility to respiratory infections in the course of congenital heart diseases, this method should be applied whenever a child experiences frequent infections. Also, abnormal radiograms should be an indication for performing an echocardiogram. A diagnosis of UAPA should be confirmed using imaging methods such as computed tomography (CT angiogram of the heart) or magnetic resonance. In case of suspected pulmonary hypertension, heart catheterization is required [8]. In both reported cases, the diagnosis was based on radiograms, echocardiograms and CT scans (Figures 1 and 2).

Unfortunately, UAPA treatment methods are limited and include revascularization (a systemicto-pulmonary shunt involving hilar arteries), pneumonectomy, lobectomy and embolization of excessively developed aorto-pulmonary collateral arteries. Also, successful re-anastomoses of peripheral pulmonary arteries and the pulmonary trunk have been described [9]. Procedures of this type, if performed during infancy, protect against many complications and allow for regular development of lungs. Early treatment may help prevent lifethreatening complications, i.e. pulmonary hypertension or hemorrhage. In case of pulmonary hypertension, a suggested therapy is comparable to the treatment of primary pulmonary hypertension and includes calcium channel blockers or antithrombotic agents. More advanced therapies with the use of bosentan, prostanoids or sildenafil [10] are becoming increasingly popular as they decrease pulmonary artery pressure and pulmonary resistance. In case of their failure, a treatment of choice is the transplantation of lungs and heart.

In the case of patients with unilateral absence of the pulmonary artery, the prognosis depends, among others, on coexistence of a heart disease, pulmonary hypertension or a combination of unilateral absence of the pulmonary artery and stenosis of the contralateral pulmonary artery.

In conclusion, unilateral absence of the pulmonary artery is a rare heart defect. Due to severe complications, thorough cardiac diagnostic tests such as echocardiogram, CT scans and, sometimes, heart catheterization are of great importance. Moreover, regular cardiac management and reliable medical care should be provided.

\section{References}

1. Bouros D, Pare P, Panagou P, Tsintiris K, Siafakas N. The varied manifestation of pulmonary artery agenesis in adulthood. Chest 1995; 108: 670-6.

2. Ten Harkel AD, Blom NA, Ottenkamp J. Isolated unilateral absence of a pulmonary artery. A case report and review of the literature. Chest 2002; 122: 1471-7.

3. Vitiello R, Pisanti C, Pisanti A, Silberbach M. Association of pulmonary artery agenesis and hypoplasia of the lung. Pediatr Pulmonol 2006; 41: 897-99.

4. Khositseth A, Siripornpitak S, Laohakunakorn P. Hypoplastic right lung associated with right pulmonary vein stenosis and systemic collateral. Congenit Heart Dis 2010; 5: 76-80.

5. Kadi H, Kurtoglu N, Karadag B. Congenital absence of the right pulmonary artery with coronary collaterals supplying the affected lung: effect on coronary perfusion. Cardiology 2007; 108: 314-6.

6. Maeda S, Suzuki S, Moriya T, et al. Isolated unilateral absence of a pulmonary artery: influence of systemic circulation on alveolar capillary vessels. Pathol Int 2001; 51: 649-53.

7. Komatsu Y, Hanaoka M, Ito M, et al. Unilateral absence of the pulmonary artery incidentally found after an episode of hemoptysis. Intern Med 2007; 46: 1805-9.

8. Griffin N, Mansfield L, Redmond KC, et al. Imaging features of isolated unilateral pulmonary artery agenesis presenting in adulthood: a review of four cases. Clin Radiol 2006; 62: 238-44.

9. Hamdan MA, Meschham YAL, Najm HK. Successful onestage repair of unilateral agenesis of pulmonary artery. Pediatr Cardiol 2005; 26: 724-6.

10. Hamdan MA, Abu-Sulaiman RM, Najm HK. Sildenafil in pulmonary hypertension secondary to unilateral agenesis of pulmonary artery. Pediatr Cardiol 2006; 27: 279-81. 